Revue des patrimoines

$6 \mid 2005$

Patrimoine en situation : l'Inventaire général entre histoire et prospective

\title{
La pierre dans l'architecture rurale en Lorraine
}

Jacques Guillaume

\section{(2) OpenEdition}

Journals

Édition électronique

URL : http://journals.openedition.org/insitu/8337

DOI : 10.4000/insitu.8337

ISSN : 1630-7305

Éditeur

Ministère de la culture

Référence électronique

Jacques Guillaume, «La pierre dans l'architecture rurale en Lorraine », In Situ [En ligne], 6 | 2005, mis en ligne le 18 avril 2012, consulté le 30 avril 2019. URL : http://journals.openedition.org/insitu/8337 ; DOI : $10.4000 /$ insitu. 8337

Ce document a été généré automatiquement le 30 avril 2019.

\section{(c)}

In Situ Revues des patrimoines est mis à disposition selon les termes de la licence Creative Commons Attribution - Pas d'Utilisation Commerciale - Pas de Modification 4.0 International. 


\title{
La pierre dans l'architecture rurale en Lorraine
}

\author{
Jacques Guillaume
}

1 La Lorraine, avec ses formations géologiques diverses, réunit un bel échantillonnage de matériaux de construction, des calcaires meusiens réputés au granite des Vosges, en passant par les grès beiges ou roses. Si la mise en œuvre de ces roches, dont la réputation a parfois dépassé les frontières, est désormais bien connue dans l'architecture majeure, elle concerne en premier lieu, au moins sur le plan quantitatif, l'architecture vernaculaire. Dans ce domaine, le milieu rural constitue un vaste champ d'étude encore peu exploré, offrant une grande variété de savoir-faire particulièrement bien adaptés aux conditions locales et parfois ne déméritant pas de l'architecture majeure.

\section{Géologie. Du socle primaire aux formations du secondaire}

2 Constituant une auréole du Bassin parisien, la Lorraine, qui comprend les quatre départements de la Meuse, de la Meurthe-et-Moselle, de la Moselle et des Vosges, appartient en grande partie à une formation jurassique du secondaire, qui se traduit par une succession de cuestas (côtes) plus ou moins arquées comprises entre la Champagne humide à l'ouest et le massif ancien des Vosges à l'est. Ce relief de côtes constitué de formations carbonatées, entrecoupé de dépressions argilo-marneuses, résulte de l'érosion différentielle d'une série sédimentaire légèrement inclinée vers le centre du Bassin parisien.

3 En Lorraine occidentale, les reliefs de côtes les plus spectaculaires sont ceux de la Côte de Moselle (Bajocien-Bathonien) et de la Côte de Meuse (Oxfordien moyen et supérieur) qui recèlent les gisements de pierre de taille parmi les plus importants de la région. 
Figure 1

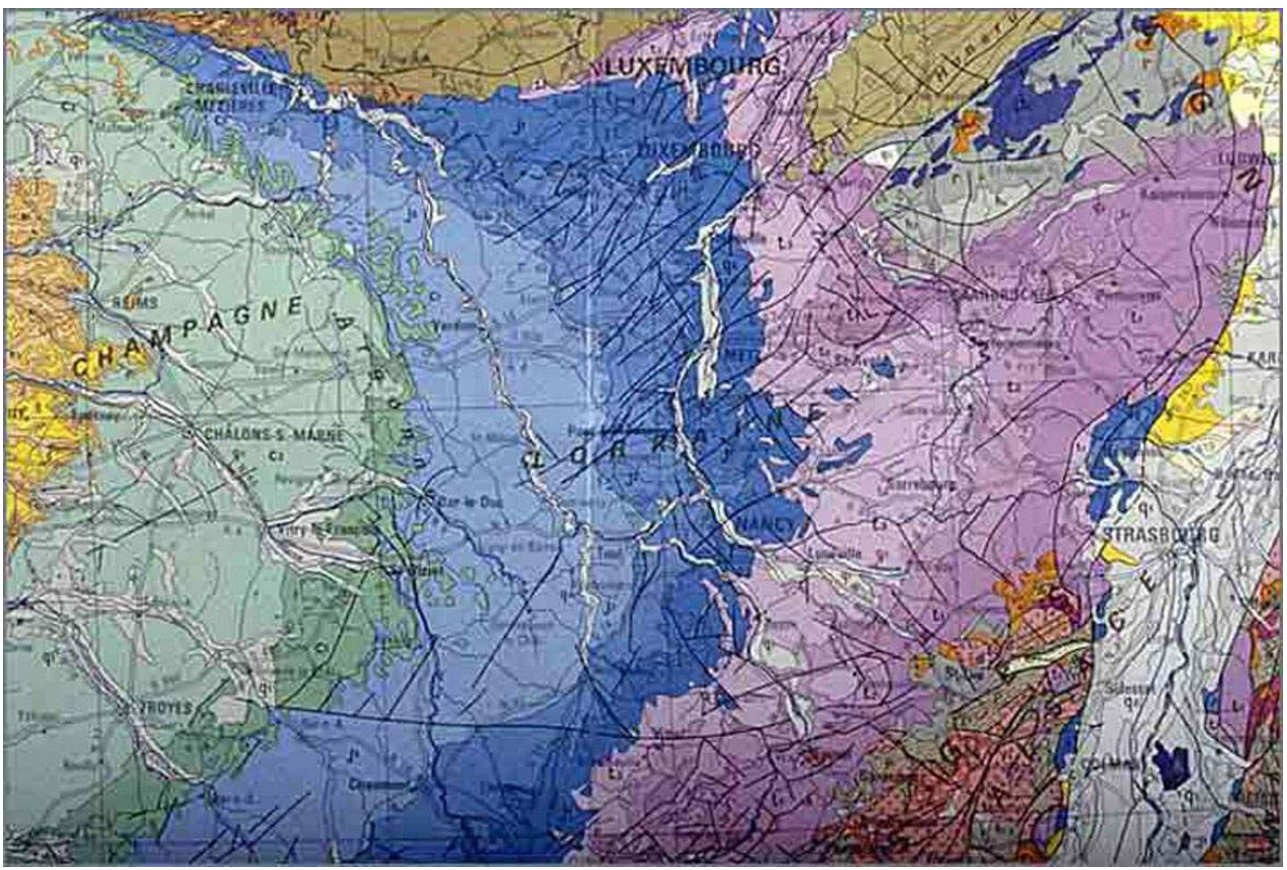

Carte géologique de la Lorraine (B.R.G.M., 1968). En bleu, les dépôts du Secondaire, en violet, les formations du Tertiaire, en rouge, le socle ancien hercynien

Repro. Inv. J. Guillaume (C) Inventaire général, ADAGP, 2004

4 A l'est, les formations calcaires laissent place aux grès vosgiens du Trias inférieur et du Lias qui s'appuient sur le socle hercynien granitique du massif vosgien (fig. $\mathbf{n}^{\circ} \mathbf{1}$ ).

\section{Les carrières et les produits}

5 Ces différentes formations ont donné lieu à une exploitation massive de la pierre dont on connaît mal les modalités de fonctionnement avant la seconde moitié du XIX siècle, faute de statistiques fiables. Le département de la Meuse, sans doute le plus riche en carrières, comptait alors 328 carrières pour un total de 665 communes. D'importance très variée, elles étaient exploitées, pour la plupart d'entre elles, de façon épisodique et ne concernaient souvent qu'un petit nombre de personnes. 
Figure 2

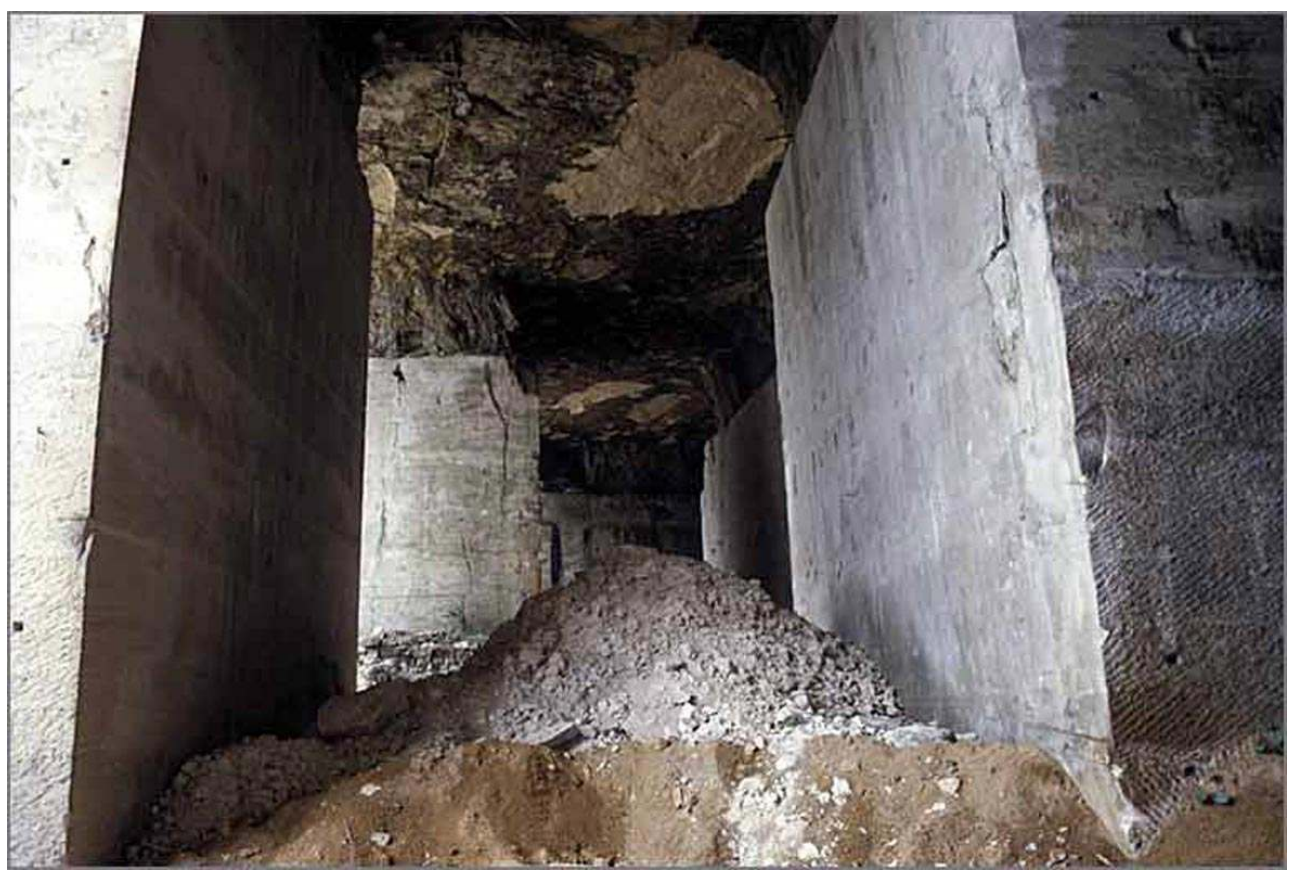

Euville (Meuse). Vue partielle des carrières souterraines

Phot. Inv. J. Guillaume (c) Inventaire général, ADAGP, 1987

6 Certaines, parfois encore en activité, occupaient jusqu'à 80 ouvriers, ainsi à Euville, d'où on extrayait annuellement près de $5000 \mathrm{~m}^{3}$ de pierre, dont une part non négligeable fit l'objet d'une exportation lointaine dès la fin du XIX ${ }^{\mathrm{e}}$ siècle (fig. $\mathbf{n}^{\circ}$ 2). Parmi les carrières les plus importantes, citons Belleray, Châtillon-sous-les-Côtes, Mécrin, Lérouville et le secteur du Perthois avec, à Savonnières, un ensemble de galeries souterraines de plusieurs centaines de $\mathrm{km}$ en longueur cumulée. Sur les 328 carrières, 140 fournissaient de la pierre de taille et 200 du moellon.

7 En Moselle, il y avait, en 1867, 253 carrières déclarées qui occupaient 727 ouvriers, les plus importantes étant à Tincry (Bajocien moyen exploité dès le XVI ${ }^{\mathrm{e}}$ siècle) et surtout Roncourt (au lieu-dit Jaumont), où travaillaient 80 ouvriers en 1866. En Meurthe-etMoselle, les carrières les plus actives se situaient à Viterne (Bajocien moyen), autour de Nancy (pierre de Balain) et à Norroy-les-Pont-à-Mousson. Cette dernière, exploitée dès l'époque gallo-romaine, était épuisée avant la fin du XIX ${ }^{\mathrm{e}}$ siècle. 
Figure 3

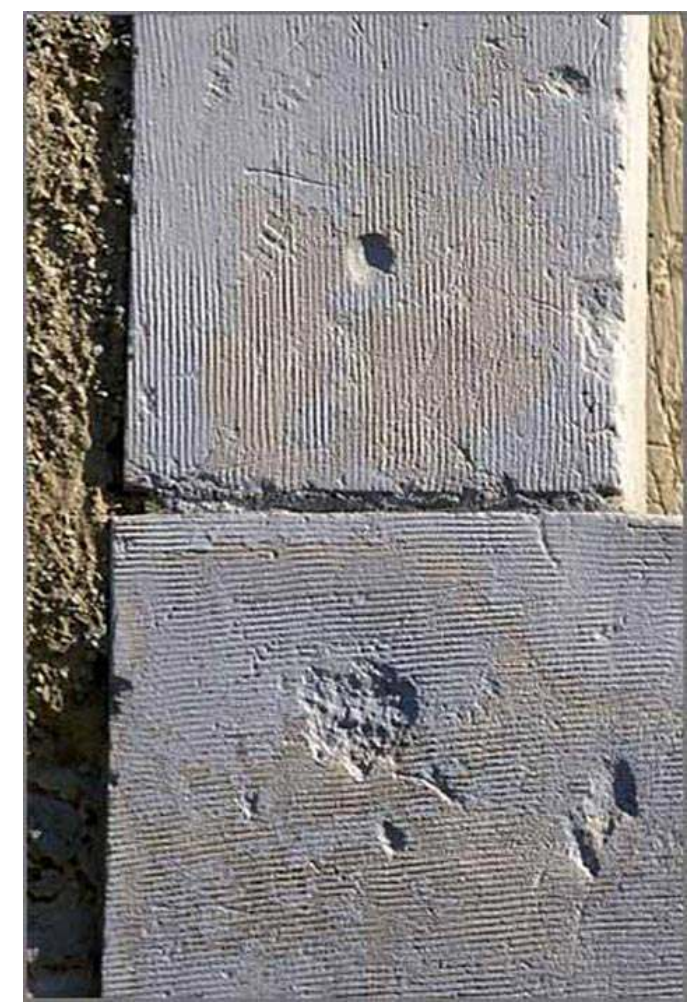

Chalaines (Meuse). Détail d'une chaîne d'angle en calcaire crayeux (XIXe siècle)

Phot. Inv. J. Guillaume (c) Inventaire général, ADAGP, 2004

Les calcaires lorrains offrent une grande variété structurelle en fonction de leur situation dans les niveaux géologiques: calcaires entroquites, oolithiques, coquilliers (bioclastiques), à lumachelles, récifaux, crayeux, sublithographiques. Leur composition et leur granulométrie se traduisent par une large gamme d'aptitudes au débitage et à la taille, ainsi que des qualités mécaniques diverses, impliquant des usages variés. C'est ainsi que les entroquites sont débitées en pierre de taille et utilisées en soubassement car elles résistent bien à la compression. Les calcaires oolithiques (pierre du Perthois, pierre de Jaumont), plus tendres, utilisables en gros œuvre malgré leur moindre résistance à l'écrasement, sont surtout propres à la sculpture car ils sont tendres. Les calcaires crayeux blancs de l'Oxfordien, sous forme de lentilles dans les bancs récifaux sont assez gélifs mais leur grain fin les a fait utiliser en encadrements de baies (Côte de Toul, région de Vaucouleurs et de Sorcy) (fig. $\mathbf{n}^{\circ}$ 3). Certains d'entre eux, comme la " pierre de Sorcy " ou de Saint-Mihiel (Meuse), ont été uniquement utilisés dans la statuaire et la confection des autels, entre le $\mathrm{XVI}^{\mathrm{e}}$ siècle et la fin du XVIII ${ }^{\mathrm{e}}$ siècle. Les calcaires à lumachelles, en revanche, sont impropres à la taille. On les employait comme parpaings ou boutisses. Les calcaires sublithographiques du Barrois sont souvent gélifs. A grain très fin, très durs et cassants, ils ont servi uniquement à la fabrication des moellons.

Les calcaires bleus de l'Hettangien (en Moselle), aux qualités médiocres, n'étaient utilisés également qu'en moellons. Les calcaires du Muschelkalk, extraits principalement à Grosbliederstroff, servaient à la fabrication de moellons et de pavés, tout comme les calcaires à gryphées du Lias d'Hettange (Moselle).

La dolomie de Brouck (Moselle), de couleur brunâtre, était débitée en pierre de taille. 
Figure 4

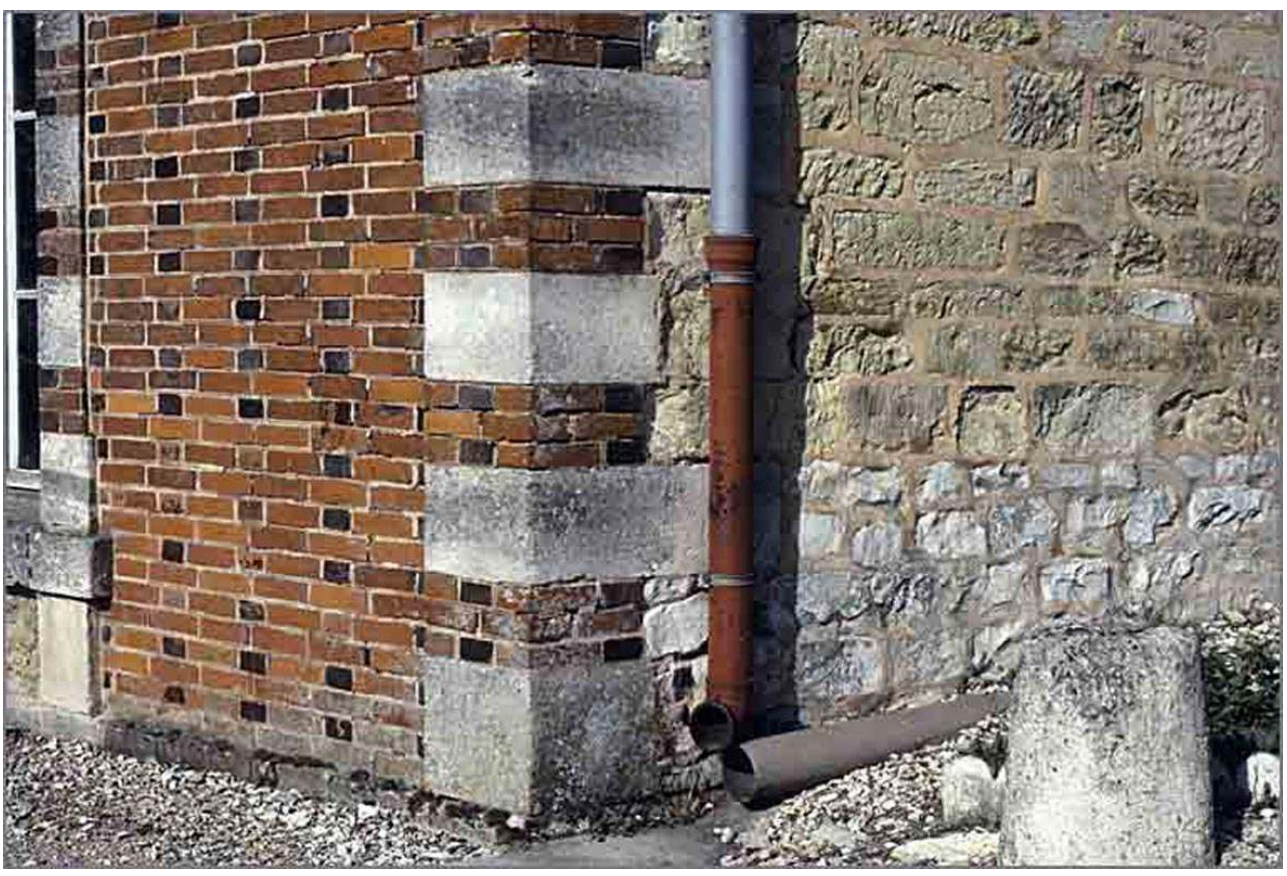

Auzéville (Meuse). Détail d'une maison rurale de la seconde moitié du XIXe siècle. A droite, mur en moellon de calcaire et de gaize, en brique à gauche

Phot. Inv. J. Guillaume (c) Inventaire général, ADAGP, 1982

11 En Argonne meusienne, a été utilisée, concurremment au pan de bois et aux calcaires locaux impropres à la taille, une roche siliceuse du tertiaire, la "gaize», de couleur verdâtre, aisée à travailler mais aux qualités mécaniques et à la résistance médiocres (fig. $\left.n^{\circ} 4\right)$.

Dans le nord de la Meurthe-et-Moselle, on importait un calcaire dur débité en plaques de faible épaisseur, dit « pierre de Mussy » (Mussy-la-Ville, Belgique), qui était utilisé comme revêtement de sol.

13 La "pierre des Ardennes ", un calcaire très dense de couleur grise à noire du Primaire (Dévonien), était exportée, au XIX ${ }^{\mathrm{e}}$ siècle, dans le nord-meusien et le Pays Haut. Sa résistance en faisait un excellent matériau, utilisé principalement dans la fabrication des éviers et des seuils de porte.

Le tuf, appelé « cron » en Lorraine septentrionale, est une formation carbonatée qui s'est déposée dans les sources. Facile à débiter et léger, il était utilisé pour les conduits de cheminée et les voûtes, comme dans bon nombre de provinces françaises.

Les quartzites de Sierck-les-Bains (Moselle), de couleur rouge violacé, réputés pour leur dureté et leur résistance à l'usure, étaient débités en pavés. 
Figure 5

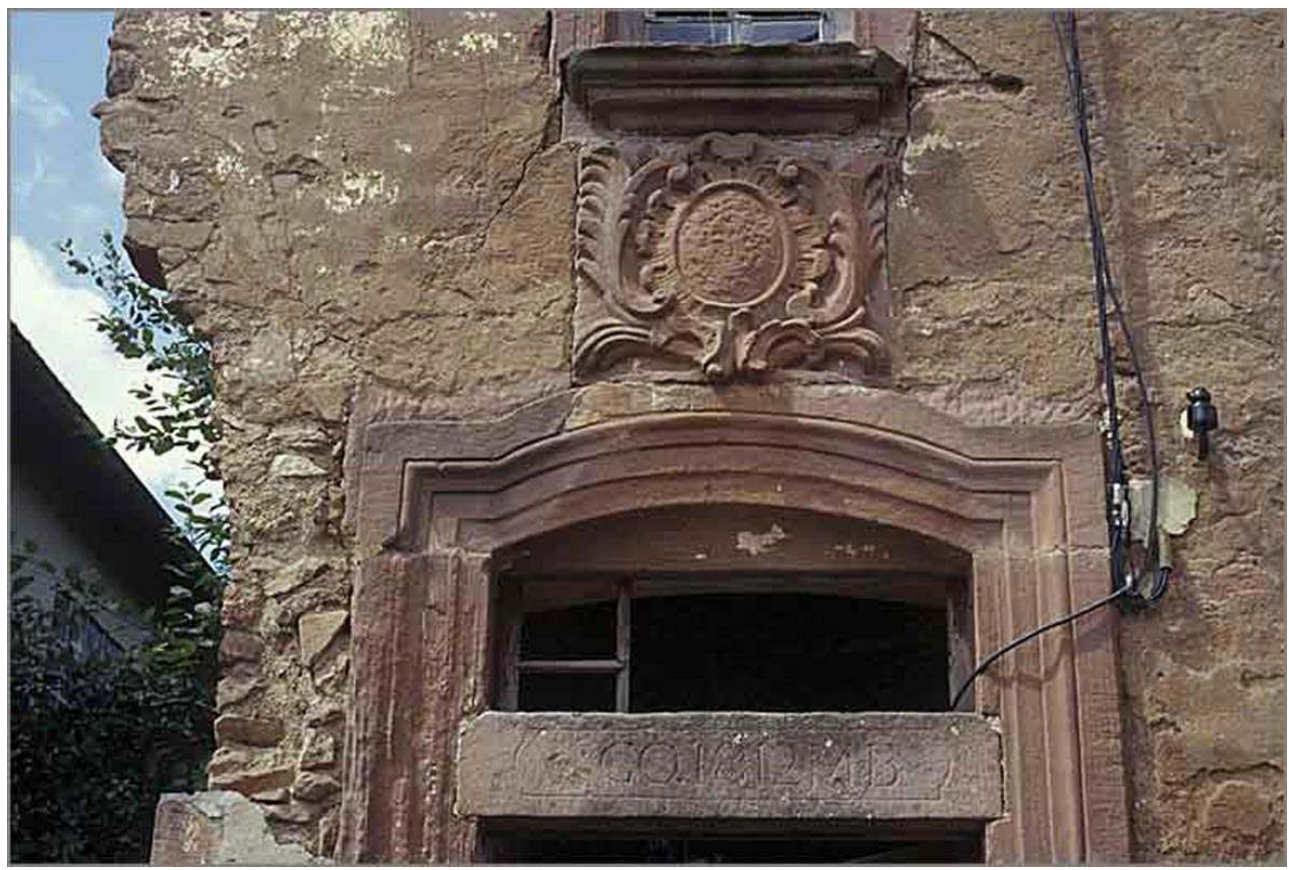

Ménil de Senones (Vosges). Détail d'une porte du XVIII siècle en pierre de taille de grès rose

Phot. Inv. J. Guillaume @ Inventaire général, ADAGP, 1996

16 Les grès bigarrés vosgiens, dont la couleur varie du gris au rose en passant par le beige, s'utilisaient aussi bien en moellons qu'en pierre de taille. Etant perméables à l'eau, on déconseillait pourtant leur emploi en fondations et parties basses de la construction, car ils sont sujets à la décomposition. Bien qu'abrasifs pour les outils et plus durs que la plupart des calcaires, ils sont parfaitement aptes à la sculpture (fig. $\mathbf{n}^{\circ}$ 5). 
Figure 6

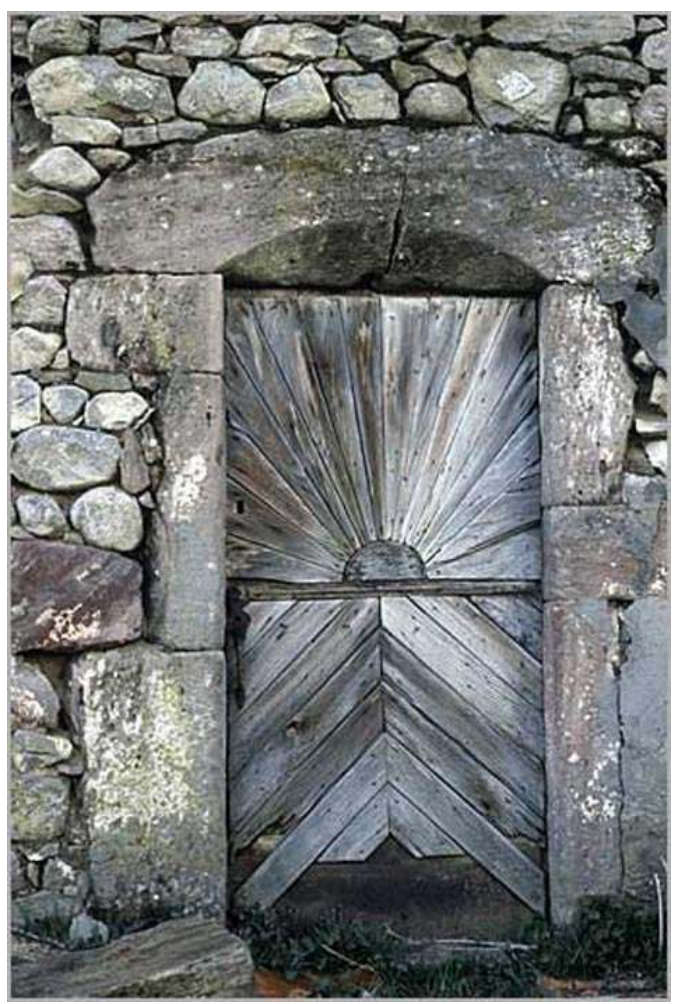

Vecoux (Vosges). Détail d'une maison rurale du XVIII siècle. Porte à encadrement de grès, mur en moellons de granite

Phot. Inv. J. Guillaume ( Inventaire général, ADAGP, 1980

17 Les granites du massif primaire vosgien, de couleur rose ou grise selon les lieux, ont été utilisés très tôt pour la construction sous forme de moellons. Ils étaient extraits sur place, au gré des besoins, à partir de rochers erratiques ou «boules d'érosion" puis, dans la seconde moitié du XIX ${ }^{e}$ siècle, en carrières, grâce à l'amélioration des techniques $d^{\prime}$ extraction et de l'outillage (fig. $\mathbf{n}^{\circ} \mathbf{6}$ ). Le granite fut utilisé de façon modeste en pierre de taille dès les années 1820-1830 (chaînes d'angle, chambranles de baies). Dans la seconde moitié du siècle, il était parfois débité en linteaux de grande taille, souvent communs à plusieurs ouvertures. Son utilisation a toujours été fortement concurrencée par le grès, à la taille plus facile et apte à la sculpture.

18 A côté des matériaux utilisés dans le gros œuvre, on doit mentionner l'utilisation, en toiture, des calcaires et des grès. 


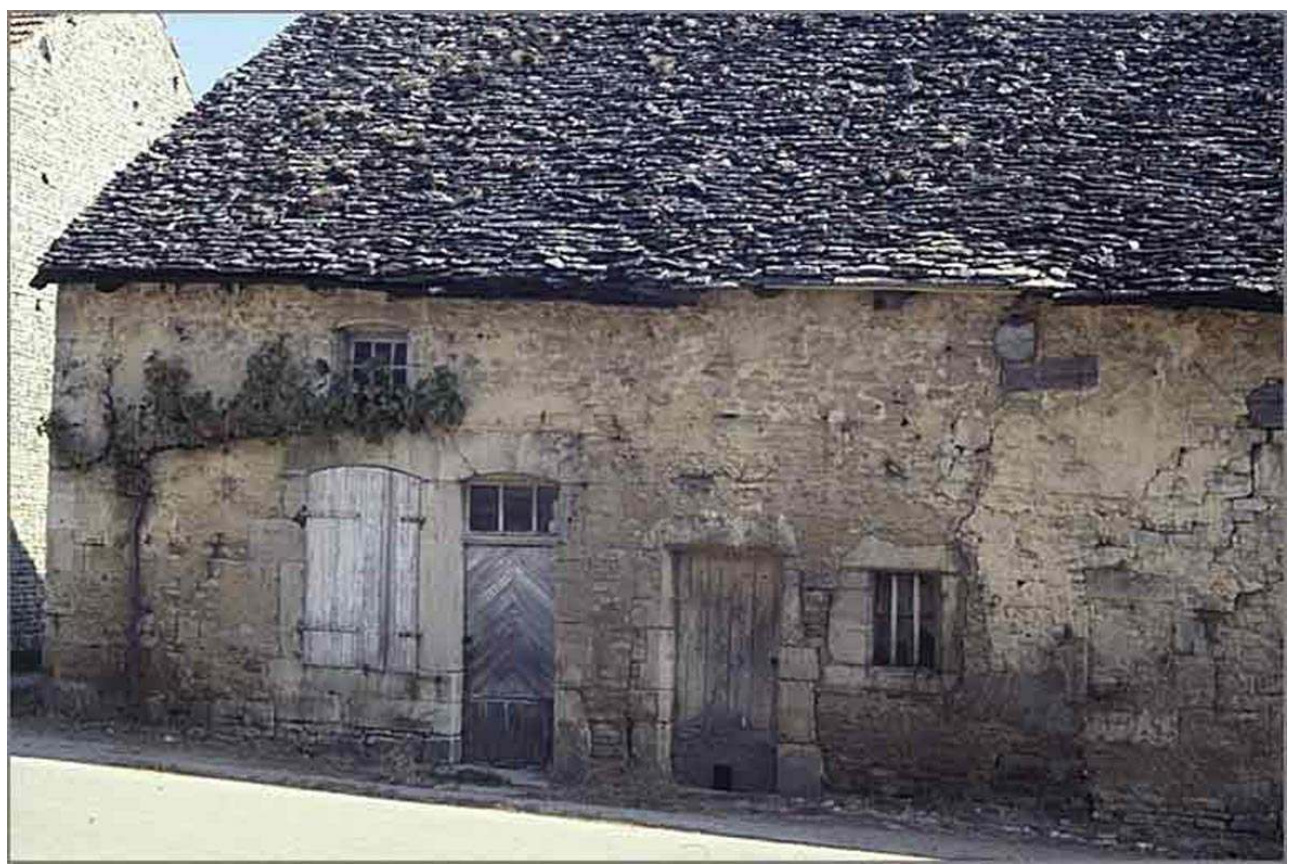

Fréville (Vosges). Détail d'une maison rurale du XVIII e siècle couverte de « laves » de calcaire

Phot. Inv. J. Guillaume @ Inventaire général, ADAGP, 1979

Les lauzes calcaires étaient extraites dans la partie occidentale du département des Vosges et dans le Sud-meusien, à la limite du département de la Haute-Marne. Il s'agit de plaques de calcaire bathonien appelées "laves", naturellement délitées et à peine équarries, extraites dans des carrières appelées «lavières ». Elles étaient posées sur le voligeage et calées avec un mortier de terre («l'herbue »). Cette technique de couverture ne fait en réalité que prolonger au nord une pratique commune en Bourgogne et en Franche-Comté (fig. $\mathbf{n}^{\circ}$ 7).

Dans le sud-est du département des Vosges, aux confins de la Haute-Saône, on a utilisé, comme matériau de couverture, entre le XVIIIe siècle et les années 1920, des dalles de grès à Voltzia liasique également appelées «laves». Extraites des bancs supérieurs de nombreuses "lavières", elles avaient naturellement tendance à se déliter horizontalement en fonction des plans de stratification. Séparées au ciseau, elles étaient ensuite soigneusement équarries. La pose se faisait à la manière des ardoises sur un lit de planchettes de bois fendu, les « ételles » ou sur un voligeage de sapin scié.

\section{Une mise en œuvre aux larges possibilités techniques}

Les sources d'archives sont peu nombreuses dans le domaine de la construction privée en milieu rural. Les quelques marchés de construction du XVIII ${ }^{e}$ siècle, s'ils nous renseignent sur l'économie générale de la construction, sont peu diserts sur le choix des matériaux et leur mise en œuvre. Ainsi, un contrat de 1717 concernant la reconstruction d'une maison des Fosses dans les Vosges, n'est guère explicite: (les maçons) « feront et tailleront et poseront les pierres de taille nécessaires audites murailles à charge qu'ils se serviront de la v(iei)lle tailles qui pourra servir suivant le traitté fait entre eux et quils les poseront aux places ou il leur sera indicqué ... » (AD Vosges, B 5477). 


\begin{abstract}
modalités du cahier des charges peuvent être appliquées avec une certaine probabilité au domaine privé. Par exemple à Folschwiller, en 1862, on exige de l'entrepreneur une pierre « extraite des bancs les plus beaux et d'un grain serré, tirée en bonne saison, sans fils, moyes, cailloux, bousin, sable graveleux et tendrières, non susceptible de geler, se déliter ou se dégrader à l'air » ou encore, à Bliesbruck, dans la construction de l'école en 1867, «le moellon ordinaire proviendra des carrières du ban et des environs, il sera de fort échantillon, d'égale dureté, tiré en bonne saison, ni gélif, ni sujet à se déliter ou à se dégrader à l'air, ébousiné à vif, bien gissant et de forme carrée ».
\end{abstract} Grâce aux recherches de Jean-Yves Chauvet dans les archives du département de la
Moselle, on est mieux renseigné sur la commande publique au XIX ${ }^{e}$ siècle, dont les

chercheur devra privilégier l'analyse sur le terrain, tout en étant conscient que des aspects aussi sensibles du patrimoine bâti que sont les enduits anciens et plus encore leurs pigments disparaissent très vite, en conséquence de la ruine des édifices et plus encore de leur « restauration » radicale.

En Lorraine, l'emploi de la pierre n'a pas toujours été exclusif. Le pan de bois, une technique de construction aujourd'hui perdue dans notre région, est encore présent dans 72 communes de Moselle et 95 en Meuse.

Figure 8

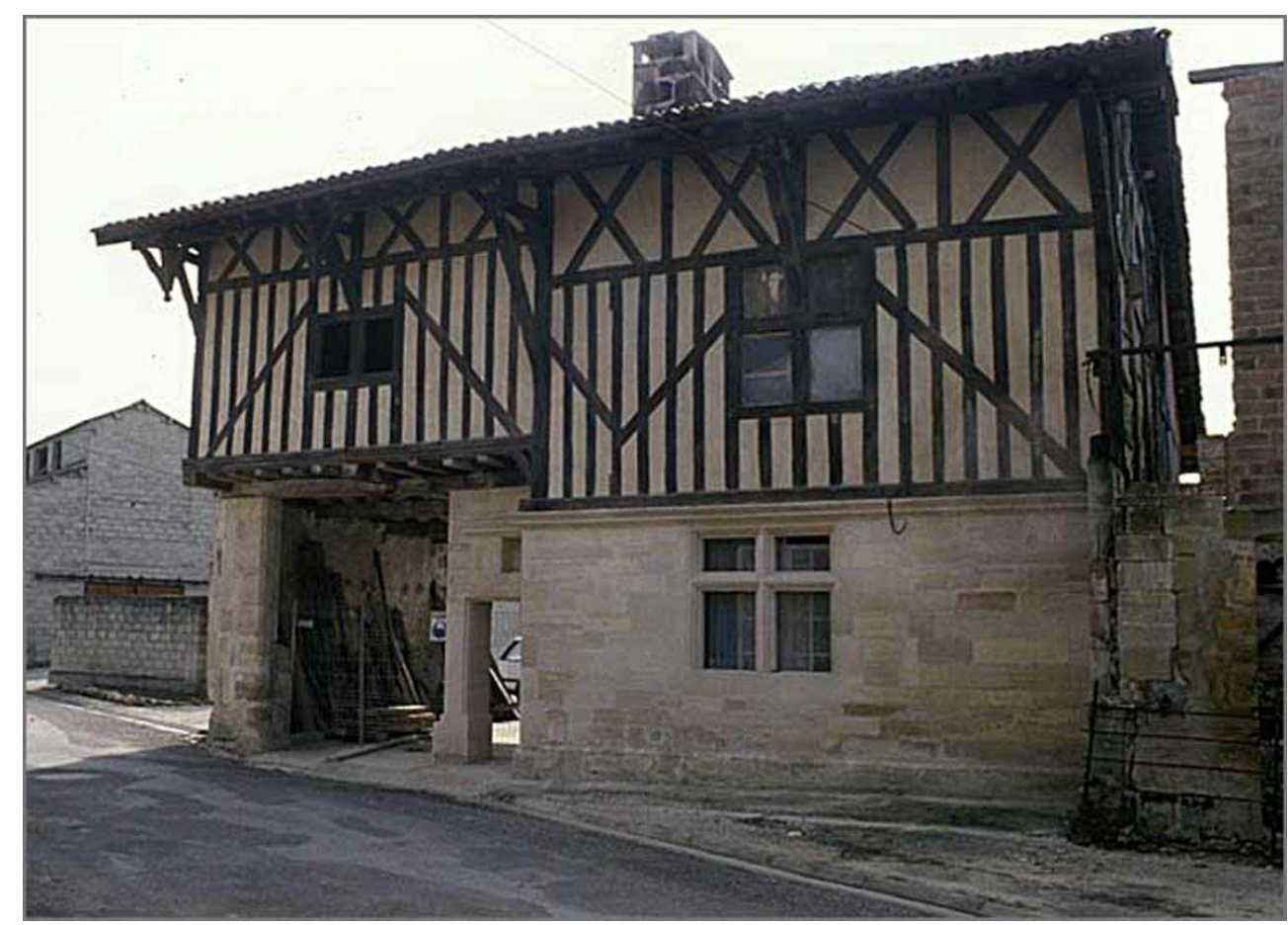

Neuville-sur-Ornain (Meuse). Maison rurale du XVIe siècle en pierre de taille du Perthois et pan de bois Phot. Inv. J. Guillaume @ Inventaire général, ADAGP, 2004

Dans le département de la Meuse, seules quelques communes argonnaises, à la limite du département de la Marne, sont concernées par un pan de bois exclusif. Les villages situés en bordure de l'Aire ainsi qu'en Basse-Argonne sont caractérisés par un habitat mixte, en pan de bois et pierre (fig. $\mathbf{n}^{\circ} \mathbf{8}$ ). La pierre, le plus souvent un moyen appareil de calcaire oolithique au nom générique de "Savonnières", concerne au moins le soubassement et le plus souvent le premier niveau des élévations. Dans les communes du Plateau barrois les 
plus éloignées de la zone de pan de bois exclusif, celui-ci disparaît du gros œuvre et se réfugie dans le cloisonnement intérieur.

Figure 9

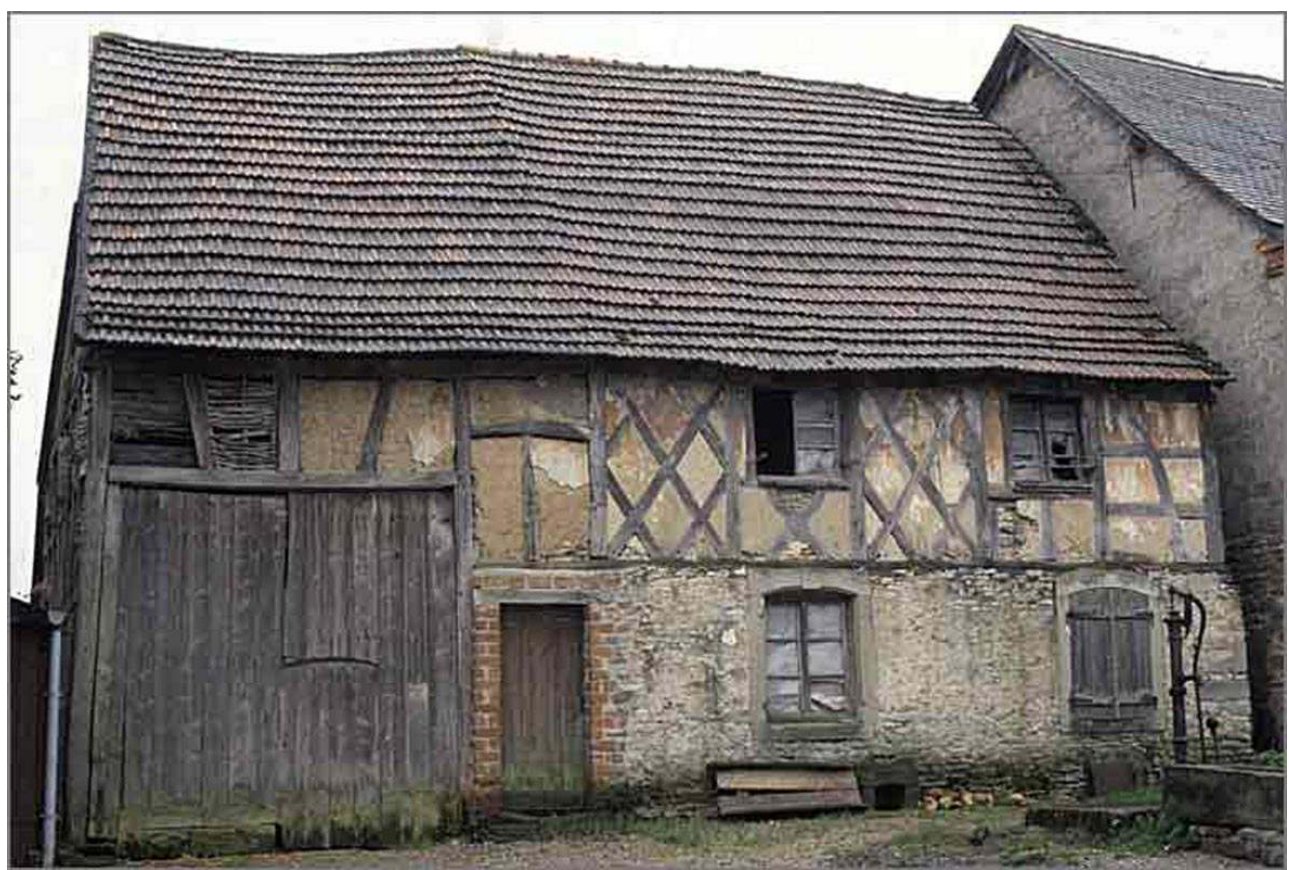

Kirviller (Moselle). Maison rurale du XVIII e siècle en moellon calcaire et pan de bois. Etat en 1982

Phot. Inv. J. Guillaume (c) Inventaire général, ADAGP, 1982

Dans l'est de la Moselle, la mixité pierre-pan de bois est le plus souvent un phénomène récent. La pierre, presque uniquement utilisée en moellon, a été substituée, peut-être dès le XVIII ${ }^{\mathrm{e}}$ siècle, au pan de bois dont les parties basses se dégradent en priorité (fig. $\mathbf{n}^{\circ} \mathbf{9}$ ). Dans les zones marginales du pan de bois en revanche, la pierre a eu tendance à supplanter dès l'origine les hourdis de terre en cloisonnement intérieur. L'usage de la pierre, un matériau inadapté au pan de bois en raison de son poids, a généralement entraîné de fortes déformations des murs de refend. 
Figure 10

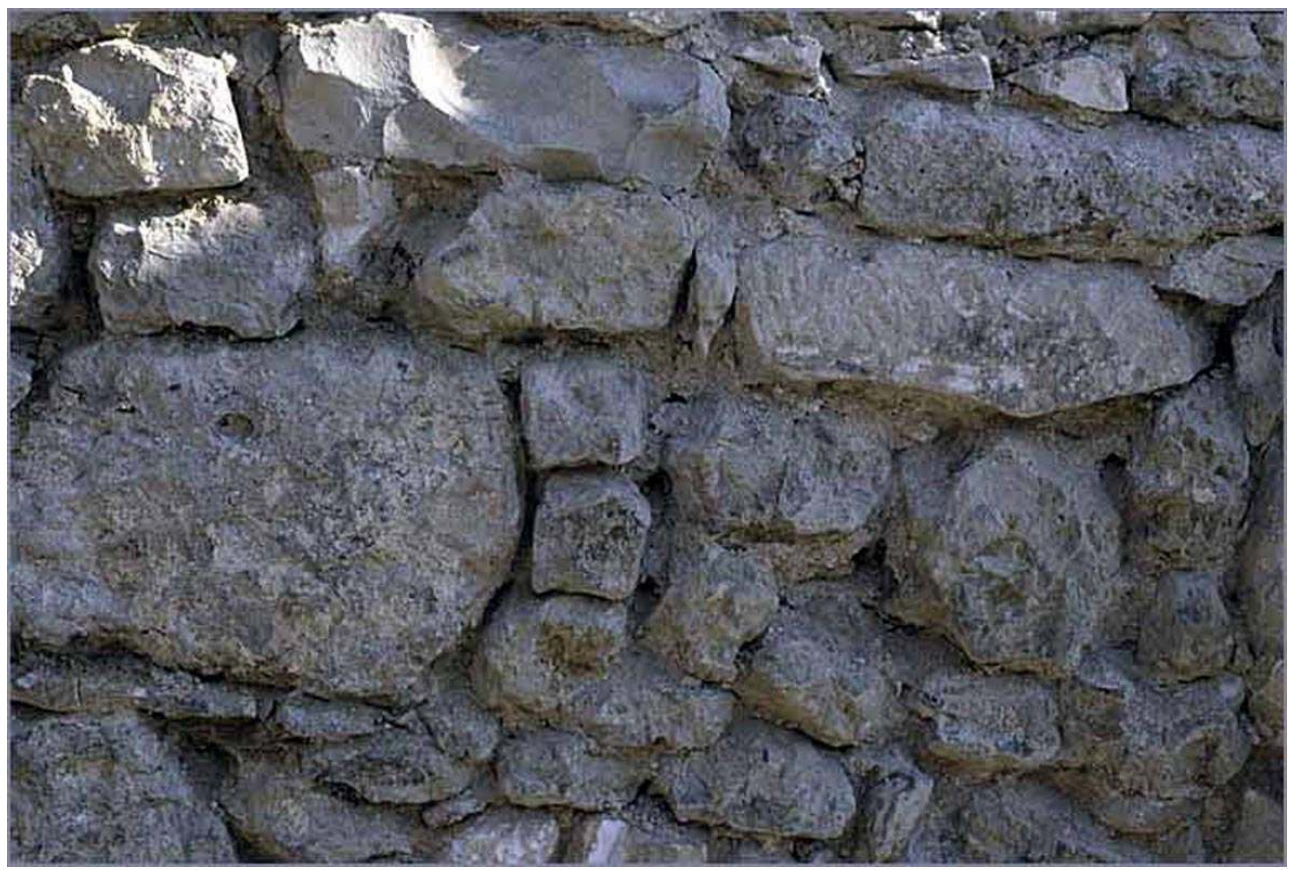

Blénod-lès-Toul (Meurthe-et-Moselle). Détail d'une maison rurale du XVIe siècle montrant, après décrépissage, une mise en œuvre de moellons grossiers à joints de mortier épais

Phot. Inv. J. Guillaume @ Inventaire général, ADAGP, 2004

27 Il va de soi que la taille du moellon était tributaire des qualités du matériau. On recherchait les bancs les plus réguliers ayant une tendance naturelle à se déliter (Sudouest du département des Vosges, Pays Haut). Parfois, la carrière ne pouvait fournir que du moellon irrégulier (granite, calcaire à lumachelles...), aussi la mise en œuvre supposait un calage avec des éclats de taille ou d'épais joints de mortier (fig. $\mathbf{n}^{\circ} \mathbf{1 0}$ ). Pour la pierre de taille, on posait les blocs en lit et non en délit pour éviter le flambage. Les faces des blocs, dressées au marteau taillant (ou rustique, grain d'orge...) étaient finies au chemin de fer, au moins pour les matériaux relativement tendres. Dans l'est de la Lorraine, le grès, après dressage des faces, était « ciselé », c'est-à-dire orné de fines stries parallèles obtenues en percussion indirecte à la chasse. Les chaînes d'angle étaient, avant la fin du $\mathrm{XVIII}^{\mathrm{e}}$ siècle, souvent finies à la boucharde, avec des ciselures relevées le long des arêtes. 


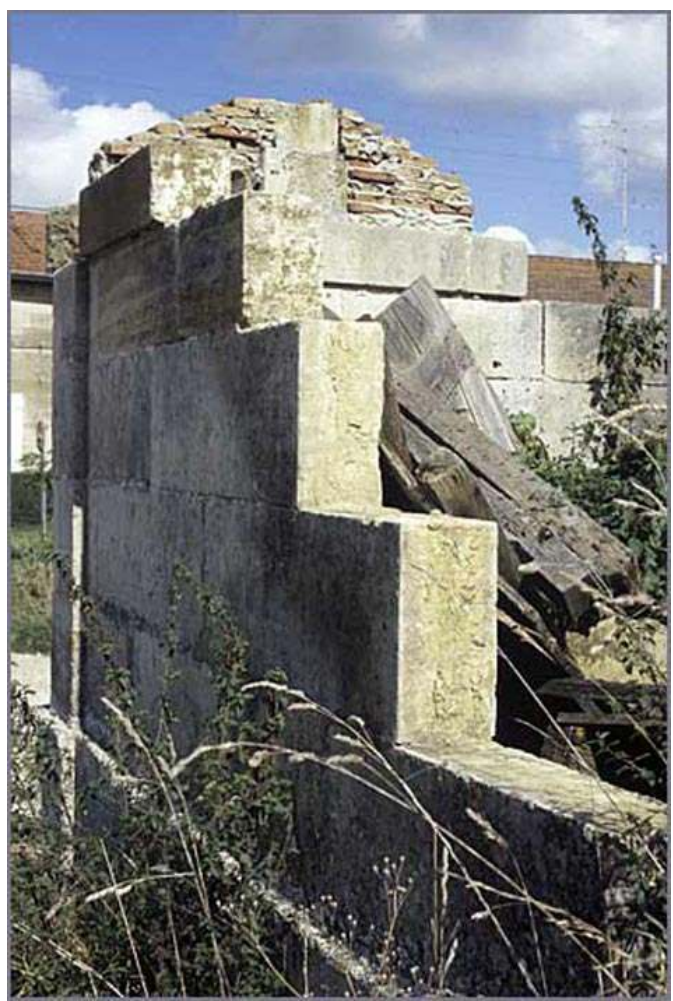

Contrisson (Meuse). Détail d'une maison ruinée du XIXe siècle construite en appareil calcaire du Perthois

Phot. Inv. J. Guillaume @ Inventaire général, ADAGP, 1982

Les mises en œuvre les plus spectaculaires concernaient les calcaires oolithiques à assises parfaitement réglées, constituées de blocs soigneusement équarris et posés en alternance. Alors que dans le Pays Haut, la pierre de taille n'était utilisée qu'en parement externe, dans le Barrois, elle concernait la totalité du mur. Ici, il s'agit de calcaires du Perthois (Bononien), exploités dès l'époque gallo-romaine, bien reconnaissables, dans les constructions $\mathrm{du} \mathrm{XVI} \mathrm{X}^{\mathrm{e}}$ à la fin du XIX ${ }^{\mathrm{e}}$ siècle, grâce à leur texture fine et leur couleur allant du blanc à l'ocre clair. Ils ont fait l'objet d'un usage intense dans le Barrois, la Basse-Argonne, concurremment au pan de bois, ainsi que dans le nord de la Haute-Marne et ont été exportés dès le Moyen Age mais principalement au XIX ${ }^{e}$ siècle, jusque dans l'Aube et la Marne (fig. $\mathbf{n}^{\circ}$ 11). A la fin du XIXe siècle, ce matériau, subissant une forte concurrence de la brique pleine, a le plus souvent été réduit à un usage secondaire: chambranles de baies, bandeaux et chaînes d'angle. L'usage de ce calcaire témoigne, au moins dès le $\mathrm{XVI}^{\mathrm{e}}$ siècle, d'une parfaite maîtrise dans la taille, il est vrai facilitée par les qualités techniques du matériau, tendre et pratiquement privé de défauts. 
Figure 12

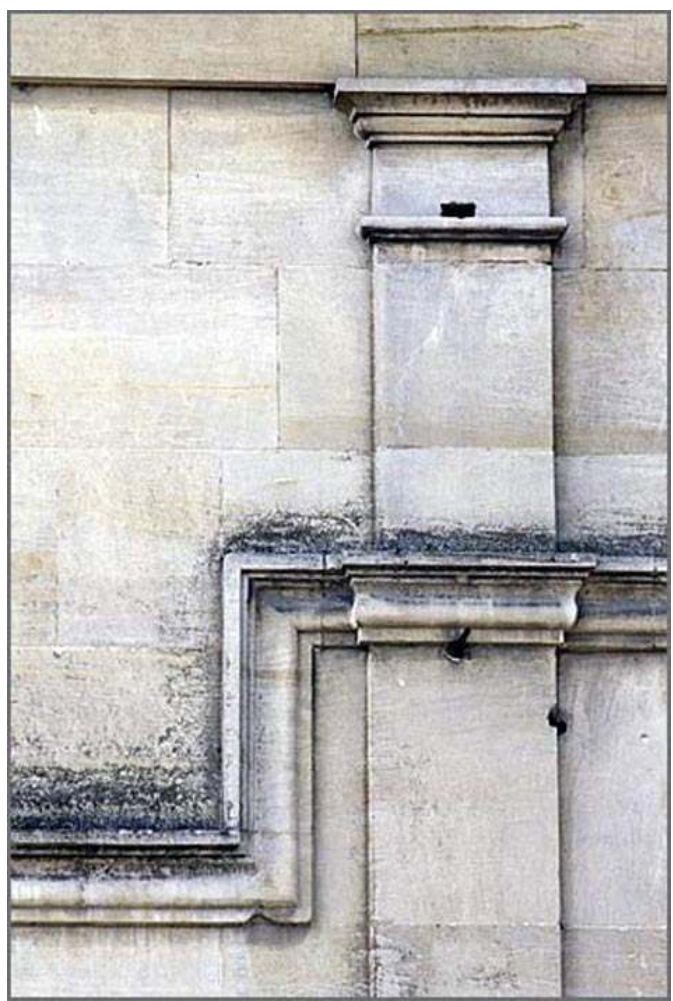

Laimont (Meuse). Détail d'une maison rurale du début du XIXe siècle en pierre de taille du Perthois. Pilastre et bandeaux moulurés animant la façade

Phot. Inv. J. Guillaume @ Inventaire général, ADAGP, 2004

Sa mise en œuvre ne pouvait se faire sans une épure commandée par le maître maçon au tailleur de pierre, qui œuvrait en carrière. La maison, que l'on peut pratiquement qualifier de préfabriquée, était sans doute rapidement construite car les blocs étaient de grandes dimensions tout en mesurant en général moins de $20 \mathrm{~cm}$ d'épaisseur. Ils étaient posés en assises parfaitement réglées sur des joints de mortier posés à bain soufflant dont l'épaisseur était inférieure à $1 \mathrm{~cm}$. Sur les tranches latérales, une gorge était ménagée afin de mieux faire adhérer le mortier. La qualité du matériau a incité les maçons, du XVI ${ }^{\mathrm{e}}$ siècle au XVIII ${ }^{e}$ siècle, à animer les façades, y compris celles de modestes fermes, de cordons moulurés offrant le plus souvent un décrochement au niveau de la porte charretière. Dès les premières décennies du XIX siècle, le répertoire décoratif s'est s'enrichi de plinthes, bandeaux, pilastres (souvent d'ordre toscan), de chaînes d'angles saillantes et de corniches moulurées (fig. $\mathbf{n}^{\circ} \mathbf{1 2}$ ). 
Figure 13

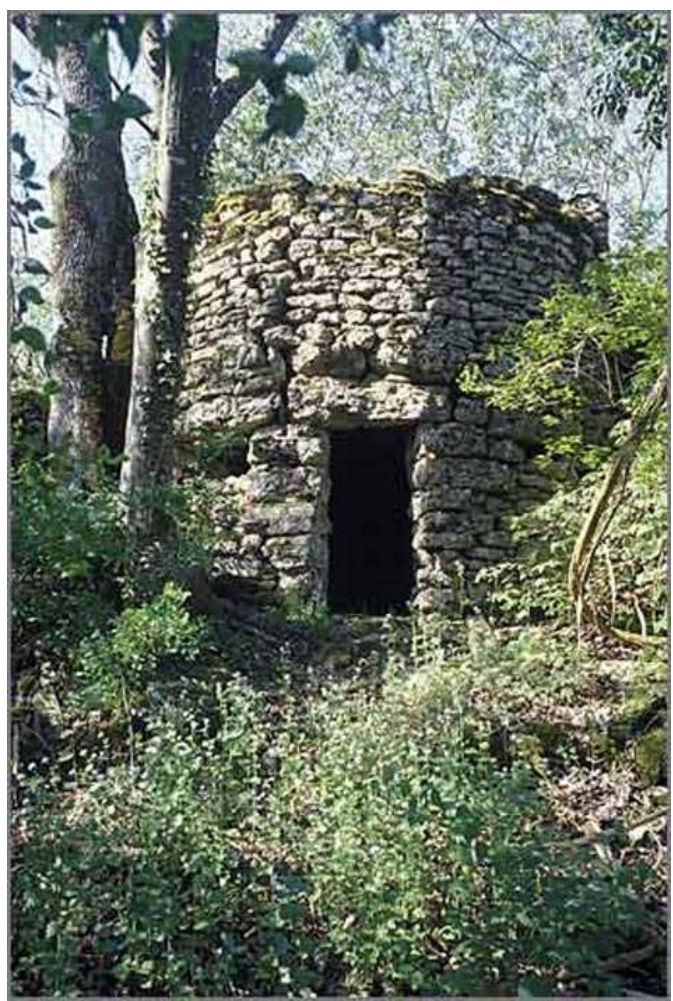

Autigny-la-Tour (Vosges). Cabane en pierre sèche

Phot. Inv. J. Guillaume ( ) Inventaire général, ADAGP, 1997

30 La construction en pierre sèche est restée très minoritaire en Lorraine. Dans le Barrois subsiste un petit nombre de constructions modestes, adossées à des murs de clôture, établis en limite de parcelles de vigne. La « cabane » d'Autigny-la-Tour (Vosges), de plan circulaire et voûtée de pierres plates clavées posées à sec, est pour l'instant un exemple unique en Lorraine, comparable aux « bories » du Vaucluse (fig. $\left.\mathbf{n}^{\circ} \mathbf{1 3}\right)$.

\section{Les mortiers et les crépis}

31 En règle générale, les mortiers, surtout en ce qui concerne la mise en œuvre des moellons, étaient constitués de terre grasse ou d'argile, parfois dégraissée par des fibres végétales, et liée, autant que faire se peut, à du sable parfois terreux, le plus souvent d'origine locale. 


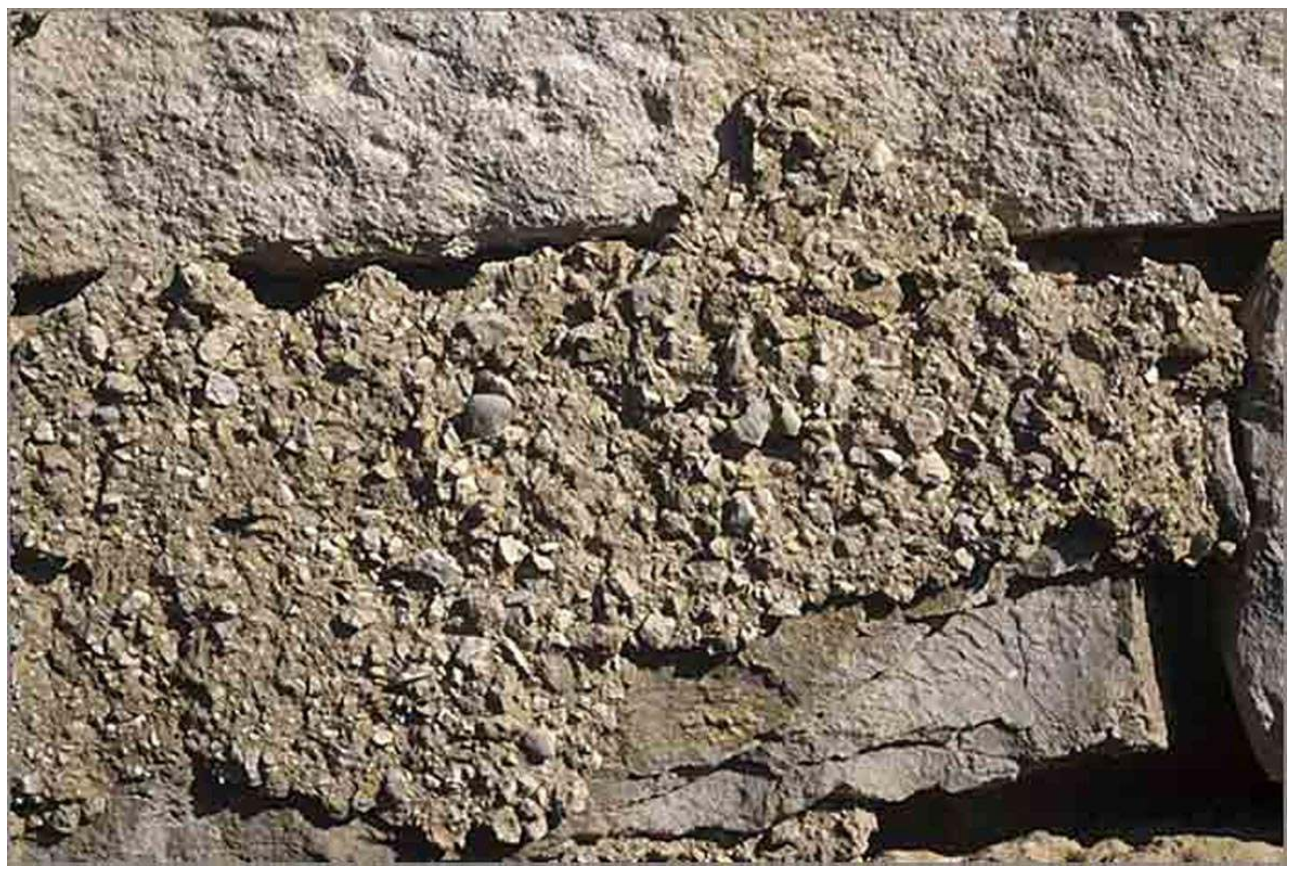

Rigny-Saint-Martin (Meuse). Vestiges d'un enduit à la « grouine » ou grèze

Phot. Inv. J. Guillaume (c) Inventaire général, ADAGP, 2004

Dans une région à forte pluviosité comme la Lorraine, les enduits protecteurs revêtent la plupart des façades, au moins celles du corps principal, à l'exception bien sûr des constructions en pierre de taille. Les annexes, en revanche, en étaient le plus souvent privées. Au XVIII ${ }^{e}$ siècle, on emploie encore des enduits terreux dégraissés avec de la bourre animale ou végétale quand le sable fait défaut. Ce dernier était extrait dans des carrières ou des sablières creusées dans le lit inférieur des rivières. Dans la Meuse, les sables de carrière sont souvent des grèzes gélifractées, ces fins éboulis de pente qui se sont formés au Quaternaire (fig. $\mathbf{n}^{\circ}$ 14). Ils ont le défaut d'être souvent terreux; dans les gisements de grès, il s'agit de sables résultant de la décomposition superficielle de ces roches; dans le massif ancien granitique, de sables grossiers issus de l'altération des arènes granitiques. Dans le Pays Haut mosellan et meurthe-et-mosellan, on substituait le plus souvent au sable du laitier de haut-fourneau concassé, appelé claine, donnant une tonalité foncée aux enduits. On rencontre également, mais de façon épisodique, de la tuile concassée dans les mortiers.

Les alluvions des rivières ont toujours été exploitées en priorité, donnant des sables naturellement lavés et débarrassés d'impuretés. C'est ainsi que le sable de Moselle, toujours très prisé, donne une tonalité rose aux crépis. La chaux aérienne, utilisée très tôt dans l'architecture majeure, tend, en milieu rural, à remplacer la terre dans le courant du XIX ${ }^{e}$ siècle, selon des modalités qui doivent encore être précisées. 


\section{Figure 15}

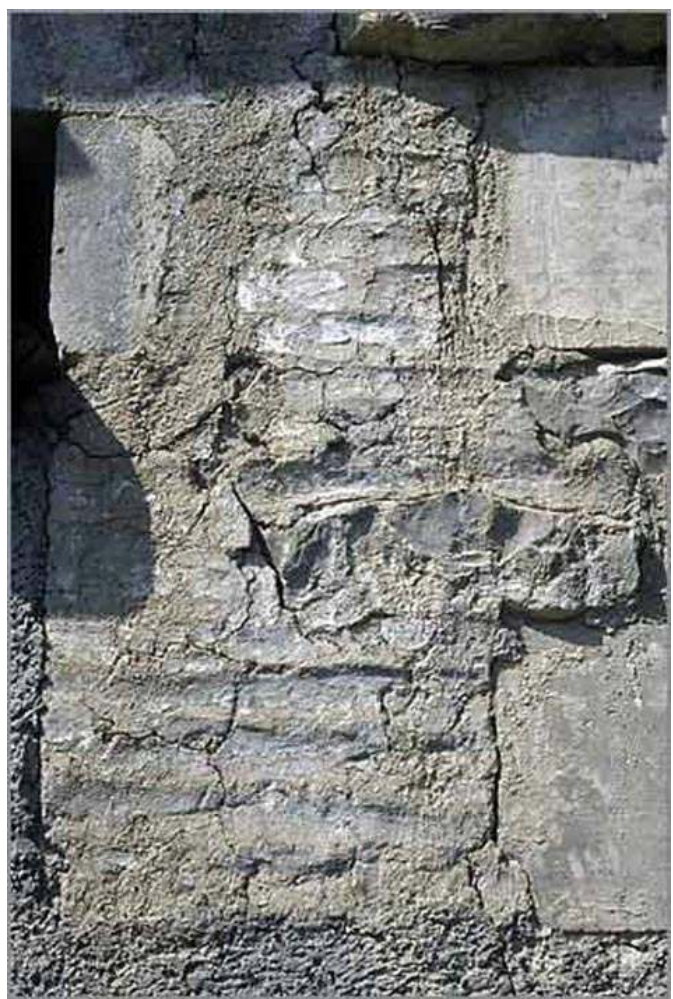

Salmagne (Meuse). Crépi à aspect ondulé, autrefois chaulé

Phot. Inv. J. Guillaume @ Inventaire général, ADAGP, 2004

Les enduits anciens, de plus en plus rares, pouvaient être à joints beurrés, et dans ce cas, relativement minces, donnant un effet plastique en fonction des irrégularités de la maçonnerie. Le plus souvent, ils étaient talochés et toujours placés au nu des encadrements en pierre de taille, jamais en saillie. Dans le Sud-meusien, le maçon donnait à la surface un aspect ondulé qui accrochait la lumière et animait la façade (fig. $\left.\mathbf{n}^{\circ} \mathbf{1 5}\right)$. Ce traitement, dont il reste peu d'exemples, a inspiré les maçons actuels qui tentent, sans succès, de le copier en grattant l'enduit frais à la truelle.

Quand la granulométrie du sable, surtout dans le Sud-meusien, ne permettait pas d'obtenir un bel état de surface, le crépi était simplement jeté à la truelle, préfigurant les crépis apparus dans la $2^{\mathrm{e}}$ moitié du XIX $\mathrm{X}^{\mathrm{e}}$ siècle, jetés au balai puis à la « tyrolienne ». 


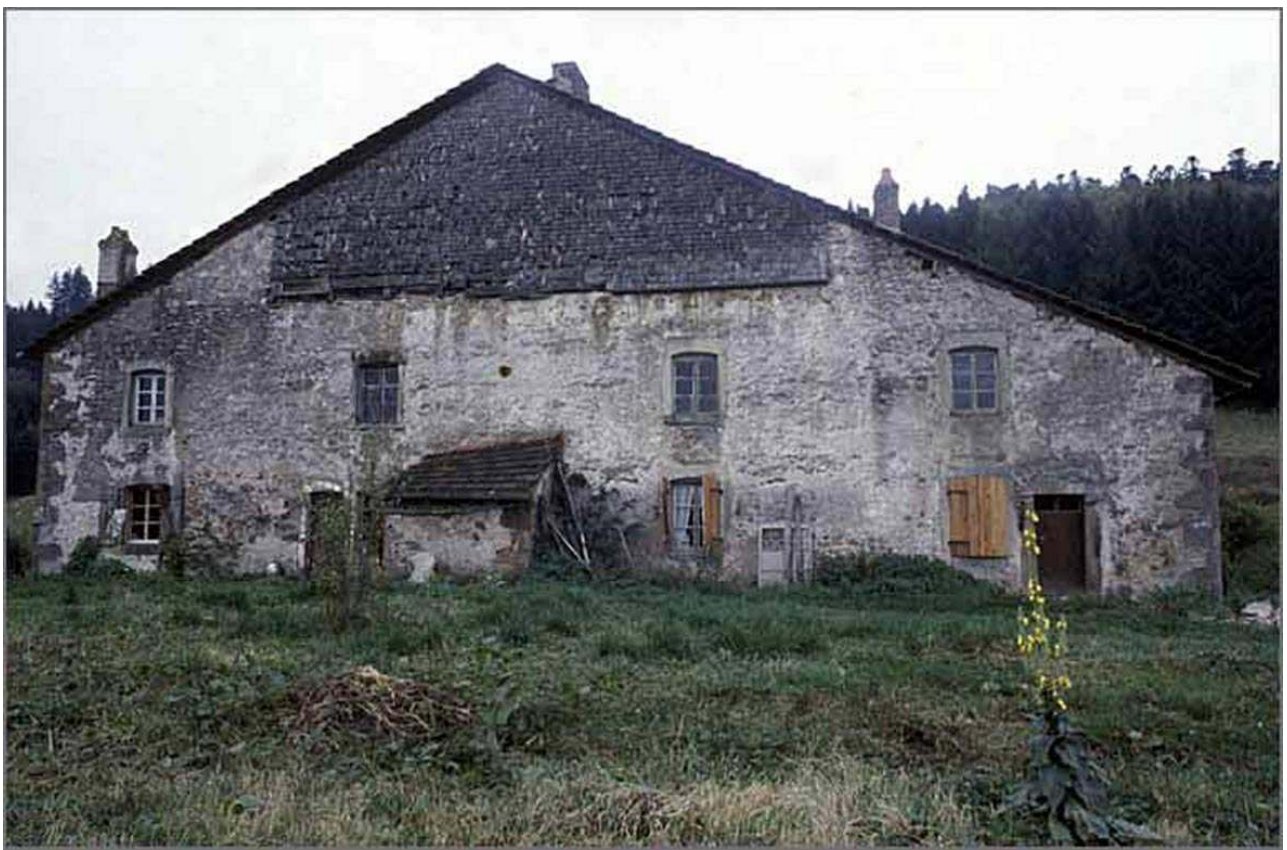

Saint-Etienne-lès-Remiremont (Vosges). Ferme de la Croisette (1777). Mur-pignon avec traces de blanchissage à la chaux

Phot. Inv. J. Guillaume @ Inventaire général, ADAGP, 1979

Dans la moitié sud de la Lorraine au moins, les enduits talochés semblent avoir été le plus souvent blanchis à la chaux, comme en témoignent les traces de badigeon qui ont été préservées en partie haute des façades, protégée par l'auvent du toit. Seule une étude poussée permettrait d'en mesurer les disparités micro-régionales. Il est d'ailleurs curieux de constater que cette pratique ne s'est maintenue que dans les Hautes-Vosges (fig. $\mathbf{n}$ -16). 
Figure 17

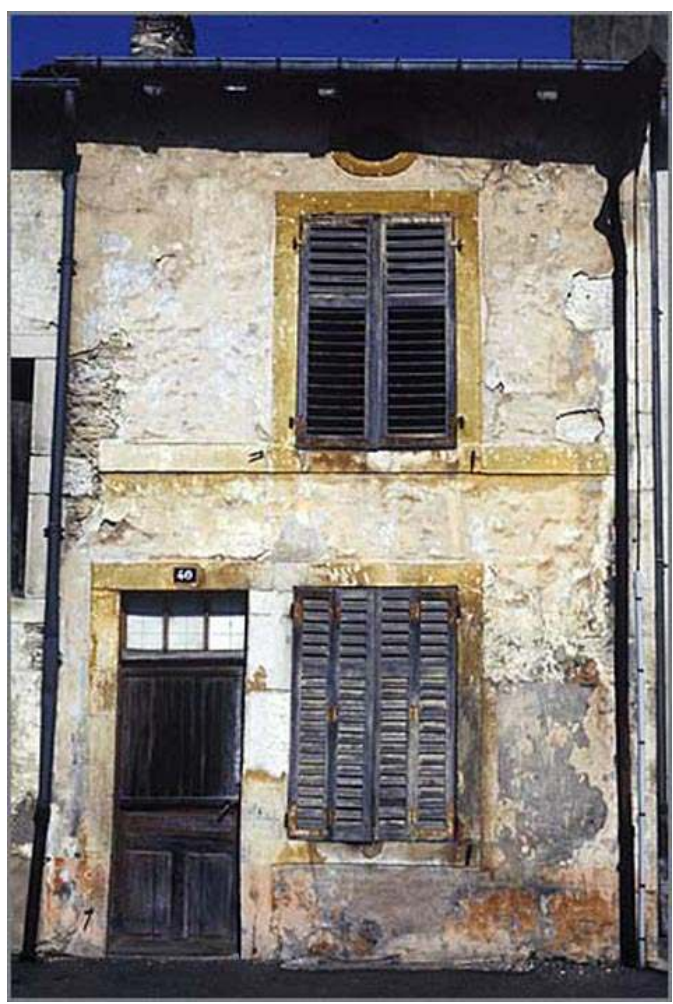

Void-Vacon (Meuse). Maison d'ouvrier à encadrements de baies peints en ocre jaune (XIXe siècle)

Phot. Inv. J. Guillaume (c) Inventaire général, ADAGP, 2004

Si les façades sont le plus souvent dépourvues de pigmentation, il n'en est pas de même des encadrements de baies, souvent badigeonnés de lait de chaux coloré grâce à des pigments minéraux. Cette pratique, qui concerne surtout l'est de la Moselle et le massif vosgien, semble avoir été la conséquence d'une influence venue des pays germaniques. Les couleurs choisies étaient en général adaptées à celle de la pierre, mais dans une tonalité différente. Les plus utilisées allaient du jaune au rouge foncé (surtout en pays de grès) en passant par l'ocre (fig. $\mathbf{n}^{\circ} \mathbf{1 7}$ ). On rencontre aussi des bleus pastel ou vifs, obtenus grâce à l'azurite (carbonate de cuivre).

En Lorraine, l'exploitation des matériaux de construction utilisés dans l'architecture vernaculaire s'est faite, jusque dans la seconde moitié du XIX ${ }^{e}$ siècle, selon deux modalités de production. 
Figure 18

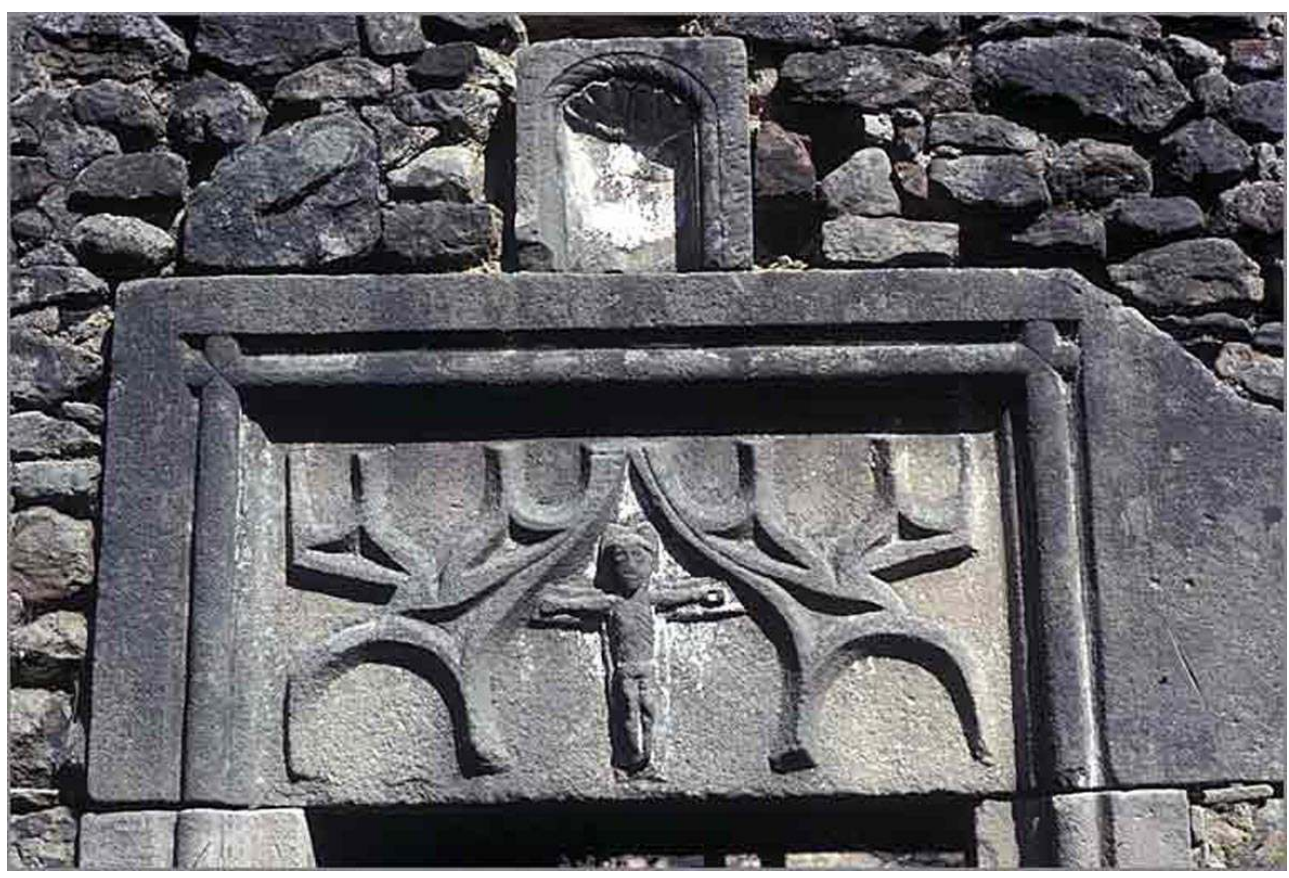

Frémifontaine (Vosges). Gros œuvre en granite et linteau de porte de la fin du XVe ou du début du XVIe siècle en grès

Phot. Inv. J. Guillaume @ Inventaire général, ADAGP, 1979

39 La première, qui répondait à des besoins immédiats peu exigeants en normes de qualité concernait des matériaux locaux, dont l'extraction était le plus souvent épisodique et sans doute pratiquée par une main-d'œuvre peu qualifiée. La seconde était adaptée à des besoins spécifiques, en l'occurrence les éléments structurants (chaines d'angle, bandeaux, corniches, encadrements de baies et aussi les éléments immobiliers par destination comme les cheminées). Or, seules quelques carrières, d'ailleurs souvent exploitées dès l'époque gallo-romaine, étaient capables de fournir les plus belles pierres. Elles ont très tôt fait l'objet d'une exploitation intensive aux mains de carriers et de tailleurs de pierre professionnels (fig. $\mathbf{n}^{\circ} \mathbf{1 8}$ ). Les produits, à forte valeur ajoutée, ont fait l'objet d'un commerce régional attesté dès le Moyen Age dans le domaine de l'architecture majeure, dès le XVIe siècle pour l'architecture vernaculaire. Il resterait à cartographier l'aire de diffusion des matériaux dans un cadre chronologique puisque, on le sait, les progrès accomplis dans les techniques d'extraction grâce à la mécanisation ainsi que l'amélioration des voies de communication ont été déterminants dans l'évolution de leur commercialisation. C'est ainsi, que dès la fin du XIXe siècle, la pierre d'Euville (Meuse) était distribuée dans plusieurs pays frontaliers. La concentration de la production, passée d'un stade artisanal à un stade industriel, aux mains de groupes d'exploitants d'envergure nationale a, conjointement avec la diffusion des matériaux nouveaux (brique, béton...) définitivement tué les savoir-faire locaux au profit de produits standardisés. 


\section{BIBLIOGRAPHIE}

Chauvet (J.-Y.) Vivre la maison lorraine. Paris, Jaher. 1981. 203 p., fig.

Gerard (C.) Architecture rurale française. Corpus des genres, des types et des variantes. Lorraine. Musée des arts et traditions populaires. Paris, Berger-Levrault. 1981. 384 p., fig.

Hilly (J.), Haguenauer (B.) Lorraine-Champagne (Guides géologiques régionaux), Masson, 1979, 215 pp.

\section{RÉSUMÉS}

La Lorraine, avec ses formations géologiques diverses, comprises entre le socle primaire et le Tertiaire, mais dominées par les dépôts carbonatés propres à cette auréole du Bassin parisien, a fourni un bel échantillonnage de matériaux de construction allant du moellon brut à la pierre de taille, dont la réputation a souvent dépassé les frontières de la France. A cette variété de ressources répondaient des savoir-faire adaptés à des besoins locaux mais aussi dignes de produits à haute valeur ajoutée, très tôt exportés hors de la région et ayant engendré des œuvres de grande qualité architecturale, y compris en milieu rural.

The Lorraine region has varied geological formations, comprised between the base strata of the primary era and the Tertiary. The main formations are of carbonated deposits however, characteristic of this zone at the rim of the Paris basin. These provide a rich selection of building stone from rubble quarry stone to cut stone. The reputation of the latter often went beyond the frontiers of the country. The variety of resources was matched by technical know-how which not only met local requirements but which could also offer high quality cut stone elsewhere. From an early date such stone was exported outside the region, and it was used in architectural works of considerable quality, not only in towns but also in the countryside.

\section{INDEX}

Mots-clés : architecture rurale, Barrois, calcaire, carrière, crépi, enduit, géologie, granit, granite, grès, Lorraine, matériau de construction, Meuse, moellon, mortier, Moselle, pan de bois, pierre, pierre de taille, pierre sèche, tuf, Vosges

Keywords : building stone, cut stone, rubble quarry

\section{AUTEUR}

\section{JACQUES GUILLAUME}

Ingénieur d'études. Inventaire. Région Lorraine. jacques.guillaume @univ-nancy2.fr 